\title{
Evaluation of a Computerized Anesthesia Report
}

\author{
Raffael Pereira Cezar Zamper ${ }^{1}$, Marcelo Luís Abramides Torres, TSA 2, Janice Leão Ferraz ${ }^{3}$, \\ Silvio Mori Neto ${ }^{4}$, Rafael Holzhacker ${ }^{5}$, Vanessa Shimada ${ }^{6}$, Maria José Carvalho Carmona, TSA ${ }^{7}$
}

\begin{abstract}
Summary: Zamper RPC, Torres MLA, Ferraz JL, Mori Neto S, Holzhacker R, Shimada V, Carmona MJC - Evaluation of a Computerized Anesthesia Report.

Background and objectives: In Brazil, the use of information systems that allows filling out anesthesia reports automatically is still in its initial stages. The objective of this study was to validate an automated anesthesia record.

Methods: This study was approved by the Ethics Commission of the institution; an industry-university partnership (Dixtal, São Paulo, Brazil and Universidade de São Paulo) was developed, and the study received a grant from FINEP (Financiadora de Estudos e Projetos do Ministério de Ciência e Tecnologia). The integration of hospital information systems for recovery of data regarding identification, preoperative evaluation, and laboratorial exams was the premise of this study. The applicability of the final version of the prototype of the automated system was evaluated by applying a semi-structured tool to 33 physicians, residents, and/or anesthesiologists during surgery procedures in 66 patients. Descriptive evaluation of the data was undertaken.
\end{abstract}

Results: The computerized system was considered reliable even for large surgeries by $81 \%$ of the participants. The majority of the anesthesiologists considered the prototype of great value for future studies and capable of meeting the requirements of anesthesia reports, bringing benefits for anesthesiologists, patients, and hospitals. The full use of the system requires training and some of its aspects can be improved.

Conclusions: Validation of this prototype of a computerized system for elaboration of anesthesia reports showed the viability of this type of solution to help anesthesiologists in their daily tasks, increasing the reliability of the data. Besides, when evaluating the applicability, anesthesiologists considered that the prototype could be useful for patients, physicians, and hospitals.

Keywords: ANESTHESIOLOGY, Organization: data recording, automated recording system.

Financial Support: Grant from FINEP - Process \# 0107027200 - Tutelage and continuing Education in Critical Care and Anesthesia.

[Rev Bras Anestesiol 2010;60(3): 285-301] CElsevier Editora Ltda.

\section{INTRODUCTION}

The need of detailed and safe documentation of medical procedures and to control health care costs have been responsible for the introduction of automated data collection and monitoring systems in several medical fields, including anesthesiology.

Received from Anesthesiology Subject and Anesthesia Division of Instituto Central do Hospital das Clínicas (HC) of Faculdade de Medicina da Universidade de São Paulo (FMUSP), SP

1. Resident; Residency Program in Anesthesiology of Hospital das Clínicas - FMUSP

2. Professor PhD of Anesthesiology from FMUSP; Supervisor of Anesthesiology from Instituto da Criança do HC/FMUSP

3. Anesthesiologist; Chief of Technical Section of Anesthesia Division from Instituto Central do Hospital das Clínicas - FMUSP

4. Dixtal company Development Asset; Graduated in Computers at Universidade Federal de São Carlos

5. Electrical Engineer at Escola Politécnica da USP; Dixtal Senior Manager of Research and Development; Master Degree in Engineer and Administration at Massachusetts Institute of Technology

6. Electrical Engineer at Escola Politécnica da USP; Dixtal Manager of Research and Development; MBA in Knowledge, Technology and Innovation at Fundação Instituto de Administração

7. Associated Professor at FMUSP; Anesthesia Division Board from Instituto Central do Hospital das Clínicas - FMUSP

Submitted on June 30, 2009

Aproved on January 20, 2010

Correspondence to:

Maria José Carvalho Carmona, M.D.

Anesthesia Division from ICHC

Av. Enéas Carvalho de Aguiar, 255, 8o andar

Cerqueira César

05403-900-São Paulo, SP

E-mail:maria.carmona@incor.usp.br
Computerized anesthesia management systems, producing an automated anesthesia form that is superior to the manual form regarding the correspondence between blood pressure levels recorded and the actual levels ${ }^{1}$, as well as other parameters monitored ${ }^{2}$, have been used in some centers for several years ${ }^{1}$ with greater sensitivity and specificity in the identification of adverse intraoperative events that are strongly related with perioperative morbidity and mortality ${ }^{3}$. On the other hand, the use of a solution that associates collection of physiological parameters and the use of drugs during anesthesia allows optimizing the evaluation of the costs of anesthesia ${ }^{4}$. This information can favor a more efficient management of consumption and storage of materials and drugs in anesthetic procedures. This type of solution also allows the creation of an automated data bank with all anesthetic records of a given service that is easier to access and improves the quality of the statistical data ${ }^{5,6}$, besides allowing the development of multicenter studies by collecting the information from centers that use the same system ${ }^{7}$.

Isolated attempts to use automated systems for data management in anesthesia with occasional introduction of relief aid can be seen in our country. Implementation of an automated system to collect data during anesthesia has been slow and gradual, dependent on prior computer knowledge of the anesthesiology team and related personnel, as well as acceptance and satisfaction of the users, which is related directly 
to the quality and versatility of the computer-based system ${ }^{8}$. The cost of the development and implementation of this solution can also restrict its applicability. After the implementation period, subjective studies have shown the superiority of the automated over the manual system, and user acceptance increases with training ${ }^{9}$.

The objective of this study was to validate a prototype of an automated anesthesia system developed by an industryuniversity partnership. Besides validating the prototype, questionnaires were applied to physicians, residents, and anesthesiologists to evaluate the applicability of the prototype.

\section{METHODS}

This study was approved by the Ethics Committee of the institution and all professionals participating in the validation phase and monitoring of the data in anesthetic procedures signed informed consents. This study was based on an industry-university partnership (Dixtal, São Paulo, Brazil, and Universidade de São Paulo), received a grant from FINEP (Financiadora de Estudos e Projetos do Ministério de Ciência e Tecnologia), and it was undertaken at the operating suite of the Instituto Central do Hospital das Clínicas da Universidade de São Paulo.

The institution that participated in this study is a tertiary level teaching hospital and, in 2005 when the data was collected it had 130 anesthesiologists and 45 anesthesiology residents. The prototype available for the second phase of validation was evaluated by 33 professionals, 29 physicians (22\% of the total) and 6 anesthesiology residents (13\% of the total). Three professionals with more than 20 years of experience in the field were invited, but they refused to participate in the study.

Validation criteria for the prototype were defined using the international standards followed by the medical equipment industry for the development of its products (IEC 60601-1-6:2004) as reference, considering the items defined as necessary by potential users participating in this study and adequate technical parameters. The main initial challenge was the viability of the data provided by the parametric monitoring system, and the project was elaborated thinking on the future integration of the prototype to existing hospital information systems for the automatic recovery of data regarding the identification, preoperative evaluation, and results of laboratorial exams.

Figure 1 shows the flow chart of the development and validation process. The first prototype created by the research and development team of the company allowed the automa-

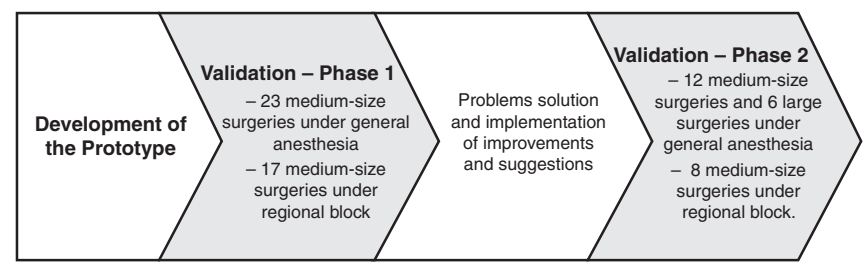

Figure 1 - Flow Chart of the Study Design. tic collection of hemodynamic and ventilatory parameters, as well as vital signs, drugs used, hydration administered, any intraoperative adverse events, and the possibility to format, store, and print an anesthesia report (anesthesia form) at the end of the procedure to be placed on the medical records of the patient. It was also possible to visualize the graphic interface of the prototype in the parametric monitor used for the patient or a notebook connected via Ethernet network, cable RS 485, TCP/IP protocol. The prototype was developed using Java technology and $\mathrm{C}++$ language. The notebook used had a $1 \mathrm{GHz}$ processor and memory of $400 \mathrm{Mb}$.

Three anesthesiologists of the hospital underwent specific training with company employees, and they helped to evaluate the prototype to allow specification of the final format of the solution.

Considering that the life cycle of the development of a technological project includes definition of requirements, development, verification of the requirements (tests), and validation during use ${ }^{10}$, the team of anesthesiologists that was trained was also involved in the definition of performance requirements and support during validation. The physicians helped the development team by suggesting more intuitive interfaces, and indicating improvements and corrections of existing functionalities to adequate the prototype to daily use. The best ways to present the data, reviewing the terms used in the system and indicating the most frequent adverse events, the drugs used more often, and pre- and intraoperative laboratorial exams requested more often, were suggested. The development team was familiarized with some clinical and documental aspects of anesthesia so the solution base developed could foresee the future integration with hospital information systems. The whole surgery scheduling process, including indication, preoperative evaluation, pre-anesthetic visit, anesthesia, and recovery period in the post-anesthetic recovery room or intensive care unit, was identified. All documentation regarding those periods, including those related to the control of restricted drugs and billing of procedures, were considered by the study group. The prototype presented here was restricted to the period of the anesthetic procedure.

During development, the prototype was used in anesthetic procedures in which one of the three physicians trained by the company and a technician of said company, who were also responsible for handling the notebook with the prototype, were always present. The anesthesiologist responsible for the patient did not use the prototype, filling out a conventional form. During four weeks, the prototype was evaluated in periodical meetings among the physicians of the team and the technicians and at the end of the fourth week the prototype of the automated system was finalized.

Once the development phase was finalized the prototype was evaluated in two phases, and some changes were made between the first and second phase to correct problems that were identified during surgeries, and improvements and suggestions were implemented.

In the first phase of the validation process, the system was used in 23 medium-size surgeries under general anesthesia 
(monitoring consisted of pulse oximeter, cardioscope, non-invasive blood pressure, and capnograph), and seventeen medium-size surgeries under regional block (monitoring the pulse oximeter, cardioscope, and non-invasive blood pressure).

The first prototype was evaluated by a team composed of four physicians of the hospital, resulting in a list with 25 suggestions for improvement, of which:

- 17 improvements of the system interface (for example: recording of non-anesthetic drugs, which were listed);

- 5 suggestions of new functionalities (for example: new registration information);

- 3 suggestions to increase the applicability of the system by the users (for example: improvement on bar code).

Twenty suggestions considered priorities were implemented along the first phase of validation and incorporated in the new prototype. At the end of this phase, a new list with 17 suggestions, of which 11 were implemented between the first and second phases of the study, was generated:

- 9 suggestions of new information or functionalities, five of which were implemented (for example: indication of the surgery);

- 3 suggestions to improve printing of the reports, which were all implemented;

- 5 suggestions to improve the interface of the system, three of which were implemented (for example: a grid on the vital signs chart).

The resulting system underwent a second phase to assess acceptability, evaluating large surgeries equivalent to what was done in the first phase, and larger surgeries to determine the acceptability of this system in those situations. In this validation, the system was evaluated in 12 medium size surgeries under general anesthesia and 8 medium surgeries under regional block using the same type of monitoring used in the first phase, besides 6 large surgeries in which invasive monitoring of systemic and pulmonary pressures were added.

In both validation phases, the anesthesiologist responsible for the case handled the system and, when necessary, with the help of a technician of the company or one of the three physicians previously trained.

To assess the applicability of this system, a questionnaire was devised specifically for this end, which would be filled out at the end of the anesthetic procedure to identify and document the point of view of the user regarding acceptance and potential contributions of the incorporation of the automated system in anesthetic procedures.

The anesthesiologists participating in the second phase of validation were invited to answer a questionnaire at the end of the anesthetic procedure. The 12-question semi-structured questionnaire was developed to identify the applicability of the automated system regarding:

- Need of prior training;

- Intuitiveness;
- Fulfilling the requirements of documentation of the anesthetic procedure;

- Practicability;

- Reliability;

- Difficulty to record the data;

- Usefulness for future studies;

- Potential benefits.

Anesthesiologists were asked to attribute a score from zero to ten for each item of the questionnaire, in which ten was the highest score.

To interpret the answers and classification of the anesthesiologists participating in the second phase of validation, parameters such as age, number of years in the field, and experience using other similar automated systems were evaluated.

Data were reported descriptively.

\section{RESULTS}

The validation process followed the recommended steps and incorporated the majority of the suggestions made during and after the first phase and before the final validation. The process was undertaken in 2005 and between the first and second phases besides incorporating new functionalities management reports were improved. In the last phase, the use in large surgeries increased the evaluation capacity of the functionalities of the system in such a way that the prototype was adequately validated.

Figure 2 shows a sample anesthesia report generated by the system, with the data captured by multi-parametric monitors (Figure 3 ) and a picture of the computer screen (Figure 4).

After the results of both phases of validation, the main tasks of inclusion of the data and generation of reports by the prototype were as follows:

- All system options are shown as a tree chart on the left side of the screen;

- Each step of the process is represented by a knot that, in turn, is divided in sub-steps also represented by knots;

- To access one option, the user should click the knot of the tree chart corresponding to that option;

- To start an Anesthesia Report, the user should register the surgical procedure in the system informing the operating room, patient, type of procedure, and date. If the patient is not registered in the system, the user should register the patient on the surgery registration screen;

- During anesthesia, the system shows the main screen with charts regarding clinical parameters, anesthetic drugs, and water and blood balance, as well as a table with other information registered in the system (notes, intercurrences, patient positioning, etc.). All information is recorded by selecting the corresponding item in the anesthesia tree chart;

- Drugs can be registered in the system through the bar code; 


\section{ANESTHESIA REPORT}

Name of the Institution

Number of the Report: 129

DATE:
DEPARTMENT: SURGERY

\begin{tabular}{lllll}
\hline NAME: & & & & NP \\
NOT DESCRIBED & & & \\
AGE: $\quad$ GENDER & HEIGHT: & WEIGHT: & BLOOD TYPE & BED: \\
$\begin{array}{l}\text { N years } \\
\text { HEALTH INSURANCE }\end{array}$ & $1.6 \mathrm{M}$ & $87.0 \mathrm{KG}$ & & 37 \\
SUS & & & \\
\end{tabular}

DIAGNOSIS

PRE-ANESTHETIC MEDICATION:

- MIDAZOLAM $5.0 \mathrm{mg} / \mathrm{mL}$

PRE-ANESTHETIC OBSERVATIONS:

Patient with hypertension treated with Higroton, Captopril;

hypothyroidism treated with Puran T4; Chest X-rays normal,

ECG normal, Laboratorial exams OK

\section{SURGICAL PROCEDURE:}

LAPAROSCOPIC CHOLECYSTECTOMY

ANESTHESIOLOGIST

Dr. Fulano

CRM

90090
ROUTE:

IM
FASTING TIME:

18 hours

BEGINNING OF THE SURGERY: END OF THE SURGERY: DURATION OF THE SURGERY: BEGINNING OF THE ANESTHESIA:

$17: 25$ 20:10

165 minutes

18:30

DESTINATION OF THE PATIENT:

PACU

CRM

99999

END OF THE ANESTHESIA: 20:25

OPERATING ROOM:

TYPE OF ANESTHESIA:
INTRAVENOUS GENERAL ANESTHESIA

DURATION OF THE ANESTHESIA:

235 minutes

RESULTS

\begin{tabular}{l|l}
\hline HOUR DESCRIPTION OF THE EVENT & HOUR DESCRIPTION OF THE EVENT \\
\hline 17:00 drug: cefazolin $1.0 \mathrm{~g}$, dose: $2.0 \mathrm{~g}$ & $17: 15$ procedure: ventilation: mechanical, controlled, volume $8000 \mathrm{ml}$, \\
17:00 drug: omeprazole $40.0 \mathrm{mg}$, dose: $40.0 \mathrm{mg}$ & pressure 18/21, rr 10, peep 7 \\
17:00 drug: metochlopramide $5.0 \mathrm{mg} / \mathrm{mL}$, dose: $10.0 \mathrm{mg}$ & $17: 20$ procedure: capnograph \\
17:00 drug: dypirone $500 \mathrm{mg} / \mathrm{mL}$, dose: $2000 \mathrm{mg}$ & $17: 20$ procedure: orotracheal intubation under direct visualization \\
17:00 drug: ketoprofen $100.0 \mathrm{mg}$ dose: $100.0 \mathrm{mg}$ & $17: 20$ procedure: ocular protection \\
17:00 drug: dexamethasone $4.0 \mathrm{mg} / \mathrm{mL}$, dose: $8.0 \mathrm{mg}$ & $17: 20$ procedure: controlled ventilation \\
17:05 procedure: equipment check & $17: 20$ procedure: orogastric tube \\
17:05 procedure: cardioscope & $17: 25$ position of the bed: head-down \\
17:05 procedure: cardioscope, nibp, spo2 & $17: 25$ position of the bed: right lateral decubitus \\
17:10 procedure: denitrogenation/pre-oxygenation & $17: 25$ beginning of surgery \\
17:10 procedure: venoclysis & $20: 10$ spontaneous ventilation \\
17:10 procedure: intravenous induction & $20: 10$ end of surgery \\
17:15 procedure: gas analyzer & $20: 25$ notes: aspirated, extubated, sent to PACU
\end{tabular}

SIGNATURE

Figure 2 - Example of the Anesthesia Report Generated by the System under Evaluation. 


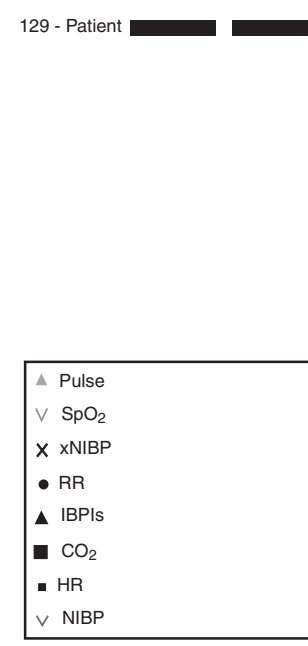

\begin{tabular}{|l|}
\hline$-3-$ FENTANYL \\
-6 - ROCURONIUM \\
-7 - LIDOCAINE \\
-1 - COMPRESSED AIR \\
-2 - OXYGEN \\
-4 - REMIFENTANLI \\
-5 - PROPOFOL \\
\hline
\end{tabular}
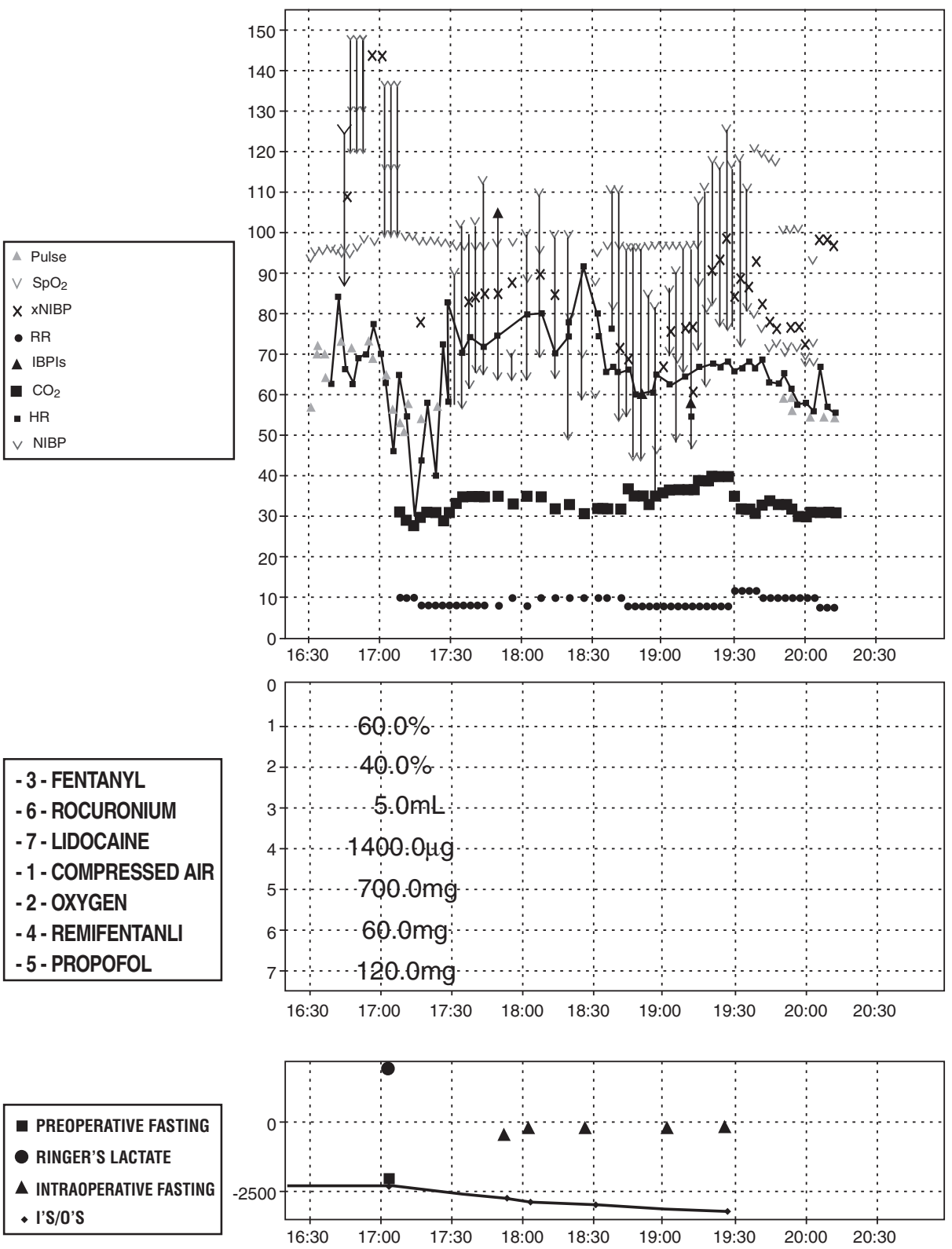

SIGNATURE

$$
\text { Dra. }
$$

CRM :

Figure 3 - Example of the Anesthesia Report with the Data from Multiparametric Monitors.

- At the end of anesthesia, the user should access the Final Report option and fill out the data to finalize the documentation after which further changes are not allowed.

At the end of this phase of the study, due to technical difficulties of the computer support team of the hospital who had other priorities at that time, it was not possible to establish the integration with the computer system of the hospital. In the future, this integration will allow testing and validating the interface that allows the registration of the surgery and other data already in the computer system of the hospital.

The mean time of experience as anesthesiologists of the professional in this study was 21.5 years. Table I shows the distribution of those professionals according to their experience in anesthesia and computer knowledge. Eighteen (55\%) 


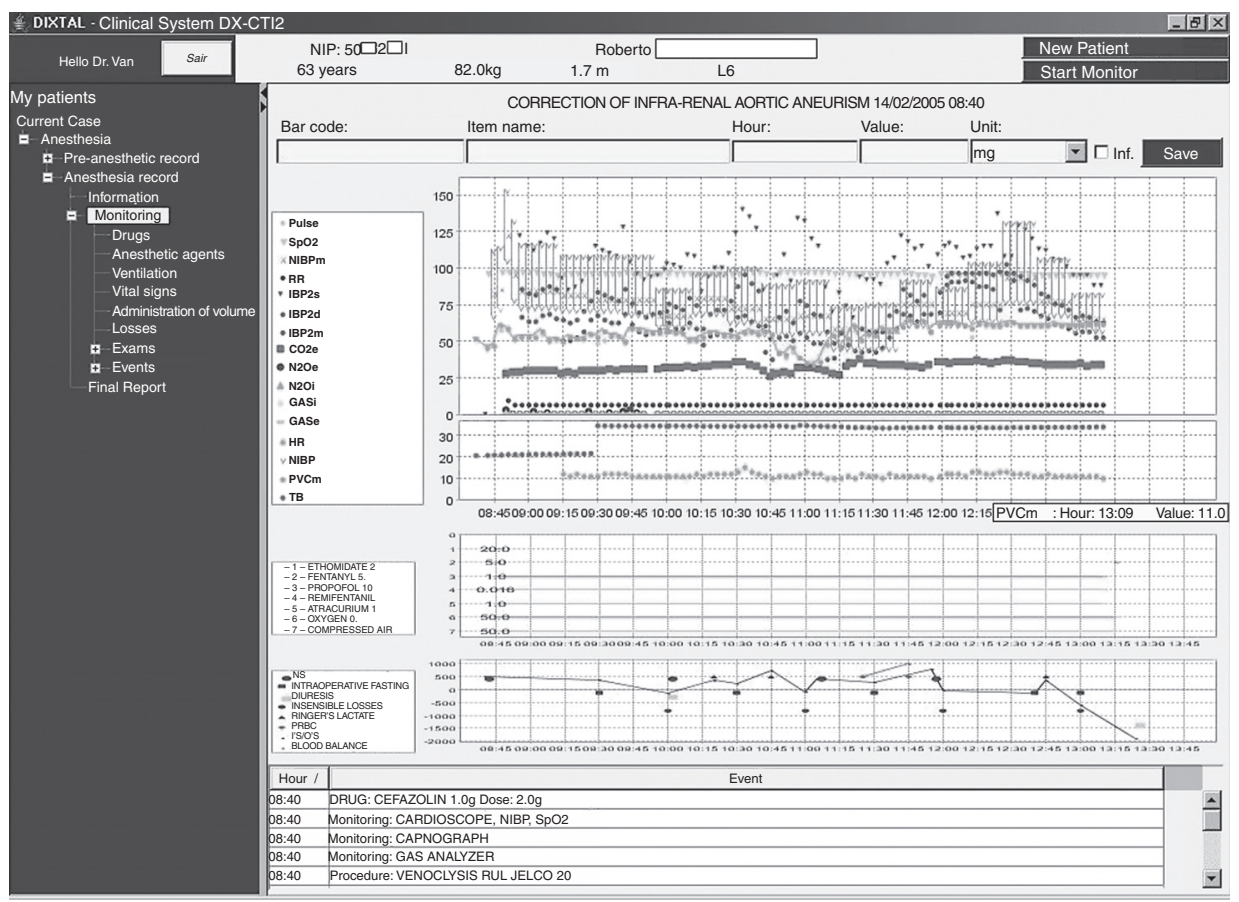

Figure 4 - Image of the Computer Screen with the Data from Multiparametric Monitors.

Table I - Distribution of Anesthesiologists According to the Length of Time in the Field and Type of Computer User2005

\begin{tabular}{|c|c|c|c|c|c|c|}
\hline \multirow{3}{*}{$\begin{array}{l}\text { Experience in } \\
\text { anesthesia }\end{array}$} & \multicolumn{4}{|c|}{ Type of computer user } & & \\
\hline & \multicolumn{2}{|c|}{$\begin{array}{c}\text { Expert or } \\
\text { Experienced }\end{array}$} & \multicolumn{2}{|c|}{$\begin{array}{l}\text { Beginner or } \\
\text { Medium }\end{array}$} & \multicolumn{2}{|c|}{ Total } \\
\hline & $\#$ & $\%$ & $\#$ & $\%$ & $\#$ & $\%$ \\
\hline$\leq 10$ years & 9 & 50.0 & 3 & 20.0 & 12 & 36.4 \\
\hline 10 to 20 years & 4 & 22.2 & 3 & 20.0 & 7 & 21.2 \\
\hline$>20$ years & 5 & 27.8 & 9 & 60.0 & 14 & 42.4 \\
\hline Total & 18 & 100.0 & 15 & 100.0 & 33 & 100.0 \\
\hline
\end{tabular}

of the professionals evaluated considered themselves to be computer experts or with considerable experience. Most professionals with less than 10 years of experience in the field - and, therefore, younger - have greater computer knowledge when compared to the group with more than 20 years of experience in anesthesia.

Among the professionals evaluated, the majority did not know any other automated system with similar characteristics. Seven $(21 \%)$ professionals reported having worked with other automated systems in anesthesia and considered themselves to have good experience with computers, and $5(15 \%)$ of them had received prior training on the use of those systems.

According to the answers of the majority of the professional, the system fulfilled their needs, and it was reliable and fast. However, 9 (27\%) professionals considered the system too slow, and 6 of them considered themselves to have good experience with computers. The majority (98\%) considered the prototype very useful for future studies (scores higher than 7). The positive evaluation of the contributions for anesthesiologists and patients was lower (73\%) than for the operating room and hospital (90\%).

The input of patient data in the system was considered easy for $30(90 \%)$ out of 33 professionals who answered the questionnaire. The inclusion of data on drugs was considered inadequate by $42 \%$ of the professionals, when the bar code was not used, and by $36 \%$, when the bar code was used. Tables II, III, and IV show the scores of the prototype regarding easiness to use, practicability, and general notes according to the type of computer user. Approximately $55 \%$ of the professionals gave scores higher than 8 regarding

Table II - Distribution of Anesthesiologists According to the Scores of Easiness of Use of the System and Type of Computer User - 2005

\begin{tabular}{lccrr}
\hline & \multicolumn{2}{c}{ Type of computer user } & \multicolumn{2}{c}{ Total } \\
\cline { 2 - 5 } Scores & $\begin{array}{c}\text { Expert or } \\
\text { Experienced }\end{array}$ & $\begin{array}{c}\text { Beginner or } \\
\text { Medium }\end{array}$ & $\#$ & \multicolumn{1}{c}{$\%$} \\
\hline 4 & 1 & 1 & 2 & 6.1 \\
5 & 2 & 7 & 9 & 27.3 \\
7 & 3 & 1 & 4 & 12.1 \\
8 & 1 & 2 & 3 & 9.1 \\
9 & 1 & 1 & 2 & 6.1 \\
10 & 10 & 3 & 13 & 39.4 \\
Total & 18 & 15 & 33 & 100.0 \\
\hline
\end{tabular}


Table III - Distribution of Anesthesiologists According to the Scores for Practicability of the system and Type of Computer User - 2005

\begin{tabular}{lccrc}
\hline & \multicolumn{2}{c}{ Type of computer user } & \multicolumn{2}{c}{ Total } \\
\cline { 2 - 5 } Scores & $\begin{array}{c}\text { Expert or } \\
\text { Experienced }\end{array}$ & $\begin{array}{c}\text { Beginner or } \\
\text { Medium }\end{array}$ & N\# & $\%$ \\
\hline 0 & 1 & 1 & 2 & 6.1 \\
2 & 1 & - & 1 & 3.0 \\
5 & 7 & 6 & 13 & 39.4 \\
6 & 1 & - & 1 & 3.0 \\
7 & 1 & 1 & 2 & 6.1 \\
8 & 3 & 5 & 8 & 24.2 \\
10 & 4 & 2 & 6 & 18.2 \\
Total & 18 & 15 & 33 & 100.0 \\
\hline
\end{tabular}

Table IV - Distribution of Anesthesiologists According to the General System Scores and Type of Computer

User - 2005

\begin{tabular}{lccrr}
\hline & \multicolumn{2}{c}{ Type of computer user } & \multicolumn{2}{c}{ Total } \\
\cline { 2 - 5 } General Score & $\begin{array}{c}\text { Expert or } \\
\text { Experienced }\end{array}$ & $\begin{array}{c}\text { Beginner or } \\
\text { Medium }\end{array}$ & $\#$ & \multicolumn{1}{c}{$\%$} \\
\hline Very good & 2 & 2 & 4 & 12.1 \\
Good & 11 & 12 & 23 & 69.7 \\
Regular & 5 & - & 5 & 15.2 \\
No answer & - & 1 & 1 & 3.0 \\
Total & 18 & 15 & 33 & 100.0 \\
\hline
\end{tabular}

the easiness of use of the system. The evaluation of the group with more experience in computers (66\%) was more positive.

Practicability had the worst evaluation with only $42 \%$ of the professionals giving scores higher than 8 and $39 \%$ gave it a score of 5 , suggesting that in this item the project needs improvement. The input of drugs is one of the aspects in need of improvement, since the input of patient data was considered very friendly for the majority $(91 \%)$ of the professionals.

The general evaluation of the prototype was considered good or very good for $81 \%$ of the professionals, and here differences between the groups with higher experience and beginners were not observed. Eight anesthesiologists (24\%) used the prototype in two or three surgeries, evaluating it in all procedures. This smaller group also showed similar distribution of anesthesiologists regarding experience in anesthesiology and computer. We observed that several items of the questionnaire had higher scores on the $2^{\text {nd }}$ or $3^{\text {rd }}$ evaluation (for example: whether training makes a difference increased from a mean of 7 to 8.25; and whether the system fulfilled their needs increased from a mean of 8 to a little over 9 , and reliability of the system went from a mean of 7 to 8.5), except for the item whether it is possible to learn how to use the system without help (intuitive system). Figure 5 shows the mean scores of the evaluations.

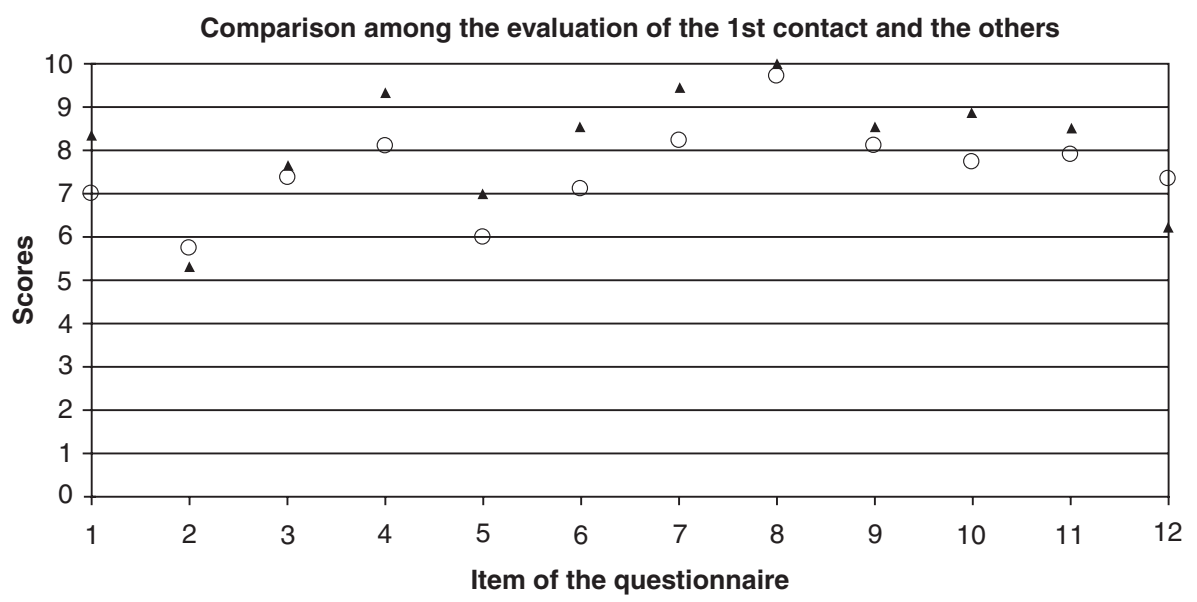

O Evaluation of the first use (1st contact)

A Evaluation on the 2nd or 3rd use.

Items of the questionnaire:

1) Does training make it easier to use?

2) Is it intuitive?

3) Is it easy to use?

4) Does it fulfill the requirements of an anesthesia report?

5) Is it practical?

6) Is it reliable?
7) Is it fast enough?
8) Can it facilitate future studies?
9) Could anesthesiologists benefit from the routine use?
10) Could the hospital benefit from the routine use?
11) Could the patient benefit from the routine use?
12) Which is the overall score for this tool on the elaboration of the
automated anesthesia report?

Figure 5 - Median of the Scores (from zero to ten) Given to the Items of the Questionnaire by the Anesthesiologists Who Used the Automated Anesthesia Report 1, 2, or 3 times. 


\section{DISCUSSION}

The positive general evaluation of the system, regardless of the computer experience, demonstrates the importance of this type of tool to record the data during the anesthetic procedure. Recovery of the information is important for health organizations, anesthesiologists, and for safety in patient care.

In the present study, designed to validate a prototype of an automated system to collect and manage anesthesia data and evaluate its applicability, the majority of the anesthesiologists considered the prototype reliable and easy to use, with a potential to facilitate collection of the data for scientific investigations and management, including in large surgeries.

In the last decades, we have observed significant improvements in surgical management of patients regarding the introduction of new anesthetics and technological development, especially regarding ventilation and monitoring of patients. However, automated systems for anesthetic documentation did not have the same development. In the USA, in 1998, only $1 \%$ of anesthesia departments used automated anesthetic documentation systems in the operating room ${ }^{11}$, and currently, this number has a tendency to be higher with the increasing use of computers in the health care. In Brazil, the use of those systems is incipient, and isolated attempts in some departments and by some companies can be observed.

The use of an automated system for data collection and management in anesthesia has the possibility of improving the care of patients undergoing any type of anesthesia. Even if intraoperative failures can be seen in those systems on collecting parameters, a bibliographic review showed that computerized records are more reliable than the manual system with conventional forms $2,12,13$. The computerized system in anesthesia could be easily accessed in the postoperative period, and intraoperative surgical or anesthetic adverse events are more easily recovered. Thus, by creating a unified data bank with the anesthesia records of a hospital, retrospective studies will be potentially more reliable ${ }^{3,6,14}$. Lesser et al. 15 investigated the incidence of bradycardia after spinal anesthesias and showed that the greatest advantages of this type of study include the reduced time for the statistical analysis, free of human errors, and the possibility to include several cases, more than 50,000 anesthetic procedures in their study.

Anesthesia departments that use those systems have better control of hospital costs ${ }^{4}$, and some centers developed studies that allowed the reduction of anesthesia costs without reducing the efficiency and quality of the service provided ${ }^{16}$. The input of drugs using the bar code showed that this functionality needs to be improved in the final version, since this is one of the key items for the safety of the patient and physician, as well for the management of resources in the operating room and hospital.

The validation proposed in this study, along with the evaluation of the applicability by using a questionnaire, is the first step for the large-scale introduction of a computerized anesthesia system in the institution where this study was carried out, and the possibility of future use in other hospitals. This endeavor contributed for the development of an electronic patient record that allows greater transparency and efficiency of health care.
Note that the involvement of physicians in the evaluation phase of the requirements to be introduced in the final version provided for a more adapted system with greater acceptance by professionals. The perception of those professionals that the system was slow showed that it should be improved constantly, especially due to the technological improvements seen every year.

We observed less adherence, and even refusal to use this tool, of anesthesiologists with more than 20 years of experience in the field. This did not reflect in the analysis of the evaluation questionnaire, since the system received high general notes from the same group with more than 20 years of experience. It should be emphasized that among the group of 14 physicians who declared to have computer experience, $36 \%$ (5) were practicing the specialty for more than 20 years. For this subgroup, using the system was easier, but they also have a higher level of requirements and critical capacity to evaluate the system.

On the other hand, it was observed that training is an important aspect, and it can be essential for the implantation of the system because the evaluation of the item refereeing to self-learning of the system received the lowest evaluation.

The difficulty of some anesthesiologists in using computerized systems, increasing considerably the time required to fill out the computerized system, was also another barrier faced. Analyzing the questionnaires, the tendency to consider difficult to learn and use the system reflects this aspect. Reference studies showed that, as a rule, $10-15 \%$ of the anesthesia time ${ }^{17}$ is spent filling out conventional forms, implying a higher chance of distraction and increased time of reaction for intraoperative events.

Although this was not investigated in the present study, since those results were not considered essential for the development of the prototype, we observed that anesthesiologists without experience with automated systems spent a long time filling out the information in the prototype of the system, but this time can be reduced considerably by the routine use of this tool and with some adaptations in the final product. Note that in the $2^{\text {nd }}$ or $3^{\text {rd }}$ contact with the system, regardless of prior experience, anesthesiologists handled the system better, which can also be observed in the results of the evaluations. On the review of the literature, little experience with computerized systems is related with greater difficulty to accept them ${ }^{18}$. Anesthesia departments with more than five years experience with those systems demonstrated, with time, that anesthesiologists showed a tendency to prefer the automated system in detriment of the manual system ${ }^{9}$.

Finally, this study detected the acceptance and viability of using the prototype of the system, through which it is possible to evaluate, at least subjectively, this tool. The questionnaire can be improved and validated in future studies, but it gave investigators a very positive evaluation of the system, since it received high scores.

To conclude, the validation of this prototype as an automated management tool demonstrated the viability of this type of solution to help anesthesiologists in their daily activities, increasing the reliability of the data, with potential benefits for patients, physicians, and hospitals. 